\title{
Mathematical Model to Optimize Production of Loading-Unloading Operations on the Vessel
}

\author{
Didmanidze Ibraim \\ Batumi Shota Rustaveli State University, BSU \\ Batumi, Georgia \\ ibraimd@ukr.net
}

\author{
Tsitskishvili Givi \\ Academic Department of General Education \\ Batumi State Maritime Academy, BSMA \\ Batumi, Georgia
}

\begin{abstract}
In scientific work it is shown, that our goal is to choose the desired option from variety of alternatives (in our case different options of loading-unloading operations on the vessel) or to take decision which is the best (optimal). Classification in this case is the grounds, as taking the choice is based on choosing certain class, which can be assigned to an alternative. Stratification and rating gives us wide option to make reasonable selection, or to take a kind of decision which will be optimal for the certain moment and occasion. These methods can be used with equal strength at all stages of the processes taking place in the area of current decision making management. This refers to the object of our study of course - solution of selecting optimal option to optimize loading-unloading operation on the vessel.

It goes without saying that variety of alternatives doesn't have any structure, thus abundance of each element was never structured randomly retrieved or no consideration has been proposed, and they are not a priority and in any order. Coming out of this it's impossible to mention which alternative is better and which is less desirable. In order to solve the task of selecting a set of alternatives successfully, it is necessary, to make structure of the given abundance of alternatives in any form.
\end{abstract}

Keywords - mathematical model, optimize production, loading-unloading operations, integer programming, linear programming.

\section{I.INTRODUCTION}

It's impossible to resolve the optimization tasks connected with cargo loading in high developing process by old methods and means. It can only be reached by using modern mathematic methods and algorithms, informational technologies and computer systems.

This process can be summed up like this: initial phase will cover all the combination model of loading-unloading operations on the vessel and acceptable route of maritime transport (in our case the vessel). Then the very optimal option will be chosen and the option will be realized, which means that the best will be chosen from the several alternate models.

In order to perceive the problem, let us discuss the obstacles we encounter when we choose the best option of transportation among the possible alternatives and what the favorable aspects of decision-making are [1].
A person in his everyday activities always takes the decision according to the situation. Sometimes this decision is acceptable, sometimes - unacceptable, thus it's false. It's noticeable to mention that the scheme of decision making process doesn't depend on the area at all, where the decision is taken. In other words, the laws of decision making are similar for any subject area, be it educational, technological process, mass service, transport shipping or any other. Decision making is nothing but the choice. Indeed, to take a decision it means to choose one specific option of activity from variety of options. Consequently, the alternative is one of the main concepts in the decision-making support system and it represents choosing alternative options.

During the shipping we choose one from several options for route selection, as it's impossible to run one and the same transport in different routes simultaneously. Besides, we choose one scheme from different loading-unloading operations, the most optimal option, as in this case it's impossible to realize several schemes simultaneously.

We don't take decision directly, but prepare the recommendations for the persons (a person) who take the decision or who need to take the decision. These recommendations are considered basic and essential condition for decision-making.

As we mentioned, the decision making is choosing. Let us make a note that we might not have variety of alternatives, from where we can choose, at the beginning. It's obvious that we should create variety of options, from where we can choose. This is the first step to resolving the problem, called the "formation of an abundance of alternatives."

It goes without saying that variety of alternatives doesn't have any structure, thus abundance of each element was never structured randomly retrieved or no consideration has been proposed, and they are not a priority and in any order. Coming out of this it's impossible to mention which alternative is better and which is less desirable. In order to solve the task of selecting a set of alternatives successfully, it is necessary, to make structure of the given abundance of alternatives in any form. 


\section{Modeling, control and information technologies -2020}

The structuring of the main types is:

* classification; stratification; Rating.

In classified variety classes are not regulated between each other, or in other words we can't say that one of the classes are more favorable (better, more expensive, cheaper, elder, etc.) than the rest. We can discuss the options of loading-unloading on the vessel, which could be classified according to any parameters. We can use the time of vessel loading, cost, cargo capacity, etc. as a parameter in this case. Obviously, if we make classification according to these parameters, then different options could be chosen in different situations for taking decision to choose the better option.

Stratification - this is division of alternative variety into levels and parts. Apart from classes strata are in good order according to any characteristics feature. In case of stratification the final choice is made between those options which are placed on the upper layer. It is worth mentioning that the strata reflect the level of quality. We could take as an example evaluation of the knowledge: the best, very good, good, average, etc.

Rating - Unlike stratification, the major role in rating plays in which "shelf" are the alternatives sorted out. One and the same ranks could be granted to the several alternatives simultaneously. In case when one and the same rank is granted to several alternatives, ranking is called soft and in case when all the alternatives are granted with the only unique number, rating is called sever.

Our goal is to choose the desired option from variety of alternatives (in our case different options of loading-unloading operations on the vessel) or to take decision which is the best (optimal). Classification in this case is the grounds, as taking the choice is based on choosing certain class, which can be assigned to an alternative. Stratification and rating gives us wide option to make reasonable selection, or to take a kind of decision which will be optimal for the certain moment and occasion. These methods can be used with equal strength at all stages of the processes taking place in the area of current decision making management. This refers to the object of our study of course - solution of selecting optimal option to optimize loading-unloading operation on the vessel.

Selection of optimal option to optimize loading-unloading operations on the vessel could be done from variety of alternatives, which is adopted by using any method. In our opinion, usage of linear and integer programming methods is most appropriate.

Simultaneous usage of linear and integer programming it's possible to process such kind of algorithm usage of which gives us opportunity to accept optimal alternatives, taking into account all its features and characteristic, as well as interactive research of certain option from these alternatives, in order to receive profitable and convenient scheme for shipment of different types of cargo.
The proposed algorithm provides automated sampling regime to load cargo on the vessel, which helps to minimize the time to load cargo. The method of branch and bound is used in algorithm, as the task requires maximizing reasonable functions in case of occasions of appropriate restrictions.

Let's put the task as follows:

Determine the optimal combination of cargo (production) to shipped on the vessel.

Let us introduce the markings in order to formalize the task [42]:

$Q_{i}, \mathbf{i}=1,2,3, \ldots, \mathbf{n}-$ type of the cargo to be loaded on the vessel;

$\mathbf{P}_{\mathbf{i}}, \mathbf{i}=\mathbf{1}, \mathbf{2}, \mathbf{3}, \ldots, \mathbf{n}-$ weight of each cargo;

$V_{i}, \mathbf{i}=1,2,3, \ldots, n$ - capacity of each type of cargo;

$\mathbf{C}_{\mathrm{i}}, \mathbf{i}=\mathbf{1}, \mathbf{2}, \mathbf{3}, \ldots, \mathbf{n}-$ cost of each type of cargo;

$\mathbf{K}_{\mathbf{j}}, \mathbf{j}=\mathbf{1}, \mathbf{2}, \mathbf{3}, \ldots, \mathbf{m}-$ maximum capacity of each type of vessel;

$\mathbf{W}_{\mathbf{j}}, \mathbf{j}=\mathbf{1}, \mathbf{2}, \mathbf{3}, \ldots, \mathbf{m}$ - maximum loading capacity of each type of vessel.

To determine optimal sum of cargo to be shipped by the vessel, we mean to choose set of cargo cost of which is optimal. It means to say, the total cost of the cargo shipped at a time should be maximum (obviously, the weight and volumes should be measures by the same values, capacity and volume are measured).

Mathematical model will look like as follows:

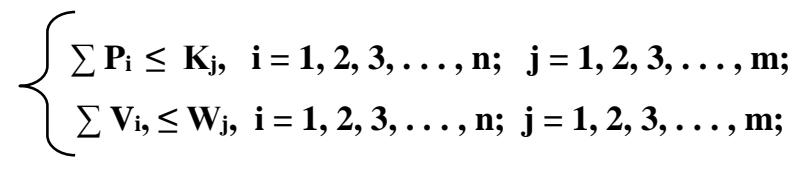

and objective function will be as follows:

$$
\mathbf{F}=\sum \mathbf{C}_{\mathbf{i}}=>\max , \mathrm{i}=1,2,3, \ldots, \mathrm{n} .
$$

Obviously, this model will target specific data for different variants of the input function (problem solution) different alternative set of values, with the aim, in the many alternatives to choose the target function of the maximum value specified constraints.

\section{REFERENCES}

[1] Didmanidze Ibraim, Tsitskishvili Givi. «Mathematical Model of LoadingUnloading Operations on the Vessel», XXVI international conference PROBLEMS OF DECISION MAKING UNDER UNCERTAINTIES (PDMU-2015). ABSTRACTS. August 24-28, 2015. Odessa, Ukraine. P. 24-25. 